\title{
Determining the Role of Food-Related Values and Food-Related Lifestyle in Influencing Food-Related Behavior of Guilford County Residents
}

\author{
Terrence Thomas $^{1 *}$, Ayodele Olofinsao ${ }^{2}$ and Kurt Taylor ${ }^{3}$ \\ ${ }^{1}$ Professor, Department of Agribusiness, Applied Economics \& Agriscience Education, C.H. Moore Agricultural Research Station, North Carolina A\&T State \\ University, 1601 East Market St., Greensboro NC 27411 USA \\ ${ }^{2}$ Graduate Research Assistant, Department of Agribusiness, Applied Economics\& Agriscience Education, C.H. Moore Agricultural Research Station, North \\ Carolina A\&T State University, 1601 East Market St., Greensboro, NC 27411 USA \\ ${ }^{3}$ Extension Associate, Coltrane Hall, College of Agriculture \& Environmental Sciences, North Carolina A\&T State University, 1601 East Market St., Greensboro, \\ NC 27411 USA. \\ *Corresponding author: Terrence Thomas, Professor A-36 C.H. Moore Agricultural Research Facility, North Carolina A\&T State \\ University, Greensboro, NC 27411, USA. \\ To Cite This Article: Terrence Thomas, Ayodele Olofinsao \& kurt Taylor. Determining the Role of Food-Related Values and Food-Related Lifestyle \\ in Influencing Food-Related Behavior of Guilford County Residents. 2020 - 8(6). AJBSR.MS.ID.001324. DOI: 10.34297/AJBSR.2020.08.001324.
}

Received: March 31, 2020; Published: 眥 May 07, 2020

\begin{abstract}
Guilford County is a food insecure county with 24 census tracts regarded as a food desert. Seventeen of these tracts are in Greensboro and seven in High point. The medical cost of combating diseases arising from unhealthy eating associated with food insecurity (practically food desert areas) is high. The objective of this study was to determine the influence of food-related values on the food-related lifestyle and food-related behavior of Guilford County residents. Data was collected by trained enumerators from a random sample of 350 respondents (82 food desert.268 non-food desert) via a telephone survey.

Data was analyzed as follows (1) factor analysis was used to identify the underlying dimensions that summarized food related value and foodrelated lifestyle, (2) cluster analysis was used to segment respondents into 2 groups each for food desert and non-food desert residents based on social values and personal values (3) following segmentation, Kruskal-Wallis $\mathrm{H}$ test was used to compare segments derived from cluster analysis on personal \&social values, food-related lifestyles, and food-related behavior (4) Kruskal-Wallis H test was used to compare food desert \& non-food desert respondents on personal \& social values, food-related lifestyles, and food-related behavior.
\end{abstract}

The result showed that food desert and non-food desert respondents share the same social values but different personal value, food desert respondents love to snack rather than eating a big dinner, prefer to buy organic food while non-food desert respondents prefer eating more than one course of dinner time and eating lunch at café restaurant. Respondents defined as food desert and non-food desert residents and the segments in each group differed on food-related values, food-related lifestyle, and food-related behavior.

Intervention programs developed should start with behaviors that are familiar to the target audience and should involve them in the design of these programs. For example, since non-food desert respondents love to eat out, educating them on how to select healthy food off the menu and start with small portion size would be more effective in modifying their behavior rather than just asking them to stop eating out.

Keywords: Cluster Analysis; Factor Analysis; Food Desert; Food Security; Kruskal-Wallis H test

\section{Introduction/Background}

Guilford County is a food-insecure county in North Carolina, USA. Twenty-four census tracts were designated as a food desert (FD) in the county [1]. An area designated as a food desert represents an extreme case of food insecurity. Access to food in food deserts is typically limited to low quality, preserved food items foods sold in convenience stores. Over time, residents of food deserts adapt to this deficient food environment, which is reflected in their eating habits and consequently their health.

In response to the reported impact on the health of food desert residents, policymakers developed measures to eradicate food 
deserts. These measures focused more on improving access to fresh fruits, vegetables, and other healthy foods. However, improving access does not always result in residents increasing their purchase of healthier offerings [2-4]. For example, the Renaissance Cooperative Grocery store that was opened to provide residents of Eastern Greensboro access to a wider range of healthy offerings closed its doors after a very brief period of operation due to low levels of patronage [5].

Getting people to eat healthier is not achievable by just providing access to healthy food, but also by addressing other factors related to eating habits such as values, and lifestyle. This study sought to assess the role of values and lifestyles in influencing eating habits. Knowledge derived from this study will prove useful in customizing intervention programs designed to address the eating habit of residents, enabling them to take advantage of the access to healthy food options.

\section{Literature Review}

In defining a food desert, factors such as location, race, socioeconomics, and access to transportation are all part of the definition [6]. Living in a food desert exposes residents to the risk of health complications resulting in high blood pressure, stroke, diabetes, heart failure, and obesity because of poor dietary decisions [7].

Improving access solves only one part of the puzzle. Other factors such as the food -related values (FRV), food-related lifestyle (FRL), and food-related behavior (FRB) of food desert residents are also important in understanding and solving the problem of poor eating habits of food desert residents.

It is suggested that FRL is the generative mechanism through which values drive behavior [8-10]. Values are the criteria people use as guidelines for evaluating stimuli [11]. They are abstract and can either be personal perspectives, group or culturally accepted norms. As an individual move through life, it is likely that his/her values change based on his/her life experiences. Food-related values are the attributes consumers look out for when making choices. They differ among people, age-group and even among family members. Just like values, food-related values are formed over time and are influenced by factors such as income, taste and, advertisement among other things.

It is assumed that lifestyle is connected to personal values. A lifestyle typically reflects an individual's values. Through lifestyle, people seek to achieve their values as expressed through various modes of action including food purchase and consumption [12]. FRL is a mental construct that explains Food-related behavior. FRL is assumed to mediate the relationship between values and FRB $[11,13]$ tested the proposed relationship. In both cases, the mediating role of FRL.

Food-related behavior sometimes referred to as Food choice is a complex phenomenon that is determined by the interactions between the consumer's psychometric traits (such as food-related values, lifestyle); the environment such as convenience, family size, social, cultural, financial factors; and the product features such as organic/ conventional, fruit \& vegetables, easy to cook \& fast food). In practice, we have multiple variables or factors operating together to influence FRB. If people do not have access to the right food, there is no opportunity to eat them and therefore, they are not likely to develop the habit of eating healthy foods. However, providing access alone without efforts to change the other factors that play a role in determining the behavior of people is also counterproductive[14,15]. This implies that policymakers should take a holistic approach in tackling the dietary related health issues associated with food insecurity/food deserts because access improvement alone will not lead to modification of behavior $[3,16]$.

\section{Materials and Methods}

Dillman's (2009) sample size formula was used to determine the adequate sample size. The sampling protocol was sent to Survey Sampling Inc. that drew a random sample proportionate to the population size of each zip code in Guilford County. A total of 10,000 telephone numbers were drawn- allocated equally among cell phones and land-line numbers considering non-working telephone numbers and businesses. The Phone numbers obtained for the survey were divided into food desert (FD) and non-food desert (NFD) residents using zip-code identifiers. 350 surveys were completed-268 from NFD residents and 82 form FD residents representing a response rate of $7.99 \%$, calculated as a percentage of the number of calls completed(350)to the total number of calls made $(4,376$.

Factor analysis with Varimax rotation was used to determine the underlying dimensions that summarize FRV and FRL to make these values and lifestyles comparable and understandable in terms of their effects on FRB [17-20]. The Kaiser-Meyer-Olkin (KMO) index was used to measure the sample adequacy or appropriateness for factor analysis. A KMO value greater than 0.5 shows the sample is adequate for factor analysis Reliability test was carried out to test the internal consistency among the set of factors making up each dimension resulting from factor analysis. Cronbach alpha value > 0.6 was considered adequate. Following factor analysis, the KruskalWallis $\mathrm{H}$ test was used to make a comparison between FD and NFD on dimensions of FRV obtained from factor analysis.

Cluster analysis was used to segment consumers into groups based on FRV using variables with the highest factor loadings in each dimension from the factor analysis result [20]. The study employed non-hierarchical clustering also known as K-means method.

The FRV scale developed by [21] was used to collect data on Guilford County's resident's FRV. The scale ranged from (1) not at all important to (10) very important. The study employed a shortened form of the instrument developed by [22] to measure FRL. The original instrument was comprised of 5 domains and 23 lifestyle 
dimensions with 69 statements. This study used 4 domains and 10 dimensions including 36 statements on a seven-point scale: (1) completely disagree, (7) completely agree to measure respondents 'response. FRB instruments was developed by [11]. It includes 37 behavioral frequency items measured on a seven-point scale: (1) never, (5) always.

\section{Results}

The results are presented and discussed below starting with FRV followed by FRL and finally FRB. The analysis will compare FD with NFD globally and compare segments of FD and NFD on FRV, FRL, and FRB.

\section{Food-Related Values}

Table 1 shows the result of the factor analysis for FRV. The factors were classified into social and personal values using Rokeach's frame work. Table 2 shows a comparison between the clusters generated from cluster analysis using variables with the highest factor loadings as the clustering variables to represent each dimension [20]. For example, in the case of FD residents "naturalness" was used to represent the "personal values" dimension and fairness represents the social dimension.

In the case of FD respondents, "fairness, tradition and convenience", were considered as representing a dimension labeled social values, while "safety, environmental impact, naturalness, and nutrition" were considered as representing personal values as shown in Table 1. In the instanceof NFD respondents, "origin, tradition, and fairness" were chosen to represent the social values dimension, while "price and convenience" were labeled the personal values dimension. Gunden \& Thomas, [23] reported a similar result in which the value dimension labeled personal (self-centered) includes naturalness \& nutrition, and the social value dimension (society-centered)includes origin, tradition \& fairness.

\begin{tabular}{|c|c|c|c|}
\hline \multicolumn{2}{|c|}{ FDable 1: Factor analysis for food-related values of a food desert and non-food desert residents of Guilford County (N=350) } \\
\hline FRV & Factor loading & FRV & Factor loading \\
\hline $\begin{array}{c}\text { Personal values Cronbach alpha } \\
0.825\end{array}$ & $\begin{array}{c}\text { Personal values Cronbach alpha } \\
0.66\end{array}$ & & 0.887 \\
\hline Naturalness & 0.825 & Price & 0.772 \\
\hline Nutrition & 0.741 & Social values Cronbach alpha 0.804 & 0.833 \\
\hline Safety & 0.73 & Origin & 0.809 \\
\hline Environmental Impact & 0.722 & Fairness & 0.804 \\
\hline Focial values Cronbach alpha 0.716 & Tradition & & \\
\hline Tradition & 0.813 & & \\
\hline Convenience & 0.746 & 0.698 & \\
\hline
\end{tabular}

1: Not at all important, 10: Very important

Table 2: Comparison between segments of food-related values by segments of food desert and non-food desert respondents of Guilford County $\mathrm{N}=350$ )

\begin{tabular}{|c|c|c|c|c|c|c|c|c|}
\hline FRV & \multicolumn{4}{|c|}{ FD } & \multicolumn{4}{c|}{ NFD } \\
\hline & SI & SII & KW & AS & SI & SII & KW & AS. \\
\hline social values & 52.42 & 11.73 & 49.93 & $0.00^{*}$ & 91.6 & 153.75 & 37.79 & $0.00^{*}$ \\
\hline personal values & 40.25 & 44.91 & 0.7 & 0.4 & 92.63 & 153.29 & 38.84 & $0.00^{*}$ \\
\hline Number of consumers & 60 & 22 & & & 83 & 185 & & \\
\hline
\end{tabular}

M represents Mean, SD represents Standard Deviation, KW represents Kruskal-Wallis $\mathrm{H}$ test, AS represents Asymp. Sig, ${ }^{*}$ signifies significant at $5 \%$, SI \&SII represents Segment I \& Segment II mean ranks respectively.

Table 2 shows that after clustering, segment 1 of FD contained 60 respondents, while segment II contained 22 respondents to give a total of 82 FD respondents. For NFD segments, segment 1 contained 83 respondents while segment II contained 185 respondents to give a total of 268 NFD respondents. The result of the comparison between the segments on FRV showed that FD segments differed only in social values. However, both segments of FD share the same personal values. NFD segments differe don both personal and social values.

\section{Food-Related Lifestyle}

Table 3 shows a summary of factor analysis results for FRL with factor loadings and Cronbach's Alpha $>0.5 .15$ items were deleted from the list of FRL because they had a weak factor loading $<0.3$ and low-reliability scores. The original FRL instrument as described above is comprised of 5 domains and 23 lifestyle dimensions with 69 statements The results summarized in (Table 3) are statements from the domain" consumption situations" and the dimension "snack vs meals" within this domain, which represent significant 
differences between FD and NFD. (Table 4) compares FD and NFD respondents globally. Table 5 shows a comparison between segments of FD and NFD. However, the results only show the dimensions in which there are significant differences.

The result from the factor analysis of FRL showing the
Cronbach alpha value and factor loadings of statements from the domain consumption situations, and dimension snack vs. meal is summarized in Table 3. This table shows only dimension in which the food desert and non-food desert respondents differ in terms of FRL.

\begin{tabular}{|c|c|}
\hline \multicolumn{2}{|l|}{ FRL } \\
\hline SNACK VS MEAL Cronbach alpha 0.669 & Factor Loading \\
\hline I eat before I get hungry, which means that I am never hungry at mealtimes & 0.774 \\
\hline I eat whenever I feel the slightest bit hunger & 0.745 \\
\hline Snacking has taken over and replaced set eating hours & 0.719 \\
\hline
\end{tabular}

Completely Disagree, (7) Completely Agree

The result of the Kruskal-Wallis $\mathrm{H}$ test presented in Table 4 shows that food desert respondents differed from NFD respondents on the domain of snacking instead of eating a full meal. This means that FD respondents prefer to eat before schedule meal time which may make traditionally scheduled meals such as lunch and dinner less important to them. In which case snacking has become their defining eating habit.

Table 4: Comparison of food-related lifestyle of food desert and non-food desert respondents of Guilford County ( $\mathrm{N}=350$ ) this table needs fixing the statements are out of alignment with the numbers.

\begin{tabular}{|c|c|c|c|c|c|c|}
\hline \multirow[t]{2}{*}{ FRL } & \multicolumn{2}{|c|}{ FD } & \multicolumn{2}{|c|}{ NFD } & \multirow[b]{2}{*}{ KW } & \multirow[b]{2}{*}{ AS } \\
\hline & $\mathbf{M}$ & SD & M & SD & & \\
\hline SNACK VS MEAL & 3.41 & 1.37 & 2.62 & 1.35 & 18.73 & $0.00^{*}$ \\
\hline I eat before I get hungry which means that I am never hungry at mealtimes & 3.48 & 2.11 & 2.73 & 1.8 & 19.05 & $0.00 *$ \\
\hline I eat whenever I feel the slightest bit hungry & 3.41 & 1.56 & 2.62 & 1.66 & 7.38 & $0.00^{*}$ \\
\hline Snacking has taken over and replaced set eating hours & 3.33 & 1.91 & 2.49 & 1.69 & 13.69 & $0.00^{*}$ \\
\hline
\end{tabular}

M represents Mean, SD represents Standard Deviation, KW represents Kruskal-Wallis $\mathrm{H}$ test, AS represents Asymp. Sig, ${ }^{*}$ signifies significant at $5 \%$.

1: Completely Disagree, 7: Completely Agree

Table 5: Comparison between segments of food-related lifestyle by respondents' segments of food desert and non- food desert respondents of Guilford $(\mathrm{N}=350)$.

\begin{tabular}{|c|c|c|c|c|c|c|c|c|}
\hline & \multicolumn{3}{|c|}{ FD } & \multicolumn{4}{|c|}{ NFD } \\
\hline & S1 & SII & KW & AS & SI & SII & KW & AS \\
\hline Price orientation & 39.82 & 46.09 & 1.13 & 0.29 & 161.45 & 102.73 & 39.75 & $0.00^{*}$ \\
\hline Freshness & 41.41 & 41.75 & 0 & 0.95 & 186.13 & 73.64 & 140.65 & $0.00^{*}$ \\
\hline Snack vs meal & 42.87 & 37.77 & 0.74 & 0.39 & 142.9 & 124.6 & 3.78 & $0.05^{*}$ \\
\hline Eating out or social event & 41.94 & 40.3 & 0.08 & 0.78 & 124.38 & 146.43 & 5.51 & $0.02^{*}$ \\
\hline
\end{tabular}

KW represents Kruskal-Wallis H test, AS represents Asymp. Sig, ${ }^{*}$ signifies significant at $5 \%$, SI \&SII represents Segment I \& Segment II mean ranks respectively.

1: Completely Disagree, 7: Completely Agree

The result of the Kruskal-Wallis $\mathrm{H}$ test shows that within FD there is no difference between segments, but within NFD segments differ on FRL as shown in Table 5.

\section{Food-Related Behavior}

Table 6 shows a summary of the result from comparing FD and NFD respondents globally on FB, while Table 7 shows the comparison between segments of FD and NFD on FRB. The results only show the FRB in which there are significant differences on FRB

The Kruskal-Wallis test result in Table 6 shows that FD and NFD respondents differ in behaviors such as buying organic foods, eating more than one course at dinner, eating lunch at café restaurants, snacking and eating big dinner.

The result of the Kruskal-Wallis test in Table 7 shows that segments of FD differed on behaviors such as eating green salad, fish, fruit, lean meat and dining at café/ restaurant; while NFD segment differed only on eating fruits.

\section{Discussion and Conclusions}

The health impact of residing in a FD has drawn the attention of policymakers both on the local and federal levels. The development of different measures to eliminate FDs through increasing 
accessibility to healthy food has not entirely been successful due to the fragmented approach to addressing the problem, which neglects to consider the psychometric characteristics of the target population. This study shows that psychometric characteristics such as respondent's s FRV and FRL influence their FRB as illustrated in Tables 2 through 7, which show:

a) There are differences between FD and NFD and between the segments of both groups on FRV.

b) The segments derived from cluster analysis based on FRV show differences on FRL and FRB.

Identifying segments can prove useful in customizing intervention programs to take advantage of differences among them to improve the efficiency of educational efforts to address eating and lifestyle habits. Instead of providing intervention programs that follow the approach of "one program fits the needs of all", it is more efficient to customize programs to target undesirable lifestyle, values and behavior by segments while building on desirable behaviors.

Table 6: Comparison of food-related behavior of food desert and non-food desert respondents of Guilford County $(n=350)$.

\begin{tabular}{|c|c|c|c|c|c|c|}
\hline FRB & \multicolumn{3}{|c|}{ FD } & \multicolumn{3}{|c|}{ NFD } \\
\hline & M & SD & M & SD & KW & AS \\
\hline I buy organic food products & 2.66 & 1.23 & 2.37 & 1.2 & 4.04 & $0.04^{*}$ \\
\hline I eat more than one course at dinner & 2.62 & 1.28 & 3.17 & 1.33 & 10.89 & $0.00^{*}$ \\
\hline I snack instead of eating a big dinner & 2.37 & 1.03 & 2.11 & 1.01 & 5.46 & $0.02^{*}$ \\
\hline I eat lunch dine at a café restaurant & 2.7 & 1.09 & 2.96 & 1.25 & 4.29 & $0.04^{*}$ \\
\hline
\end{tabular}

M represents Mean, SD represents Standard Deviation, KW represents Kruskal-Wallis H test, AS represents Asymp. Sig, SI \&SII represents Segment I \& Segment II means respectively

1: Never, 5: Always

\begin{tabular}{|c|c|c|c|c|c|c|c|c|}
\hline \multicolumn{1}{|c|}{ Table 7: Comparison between segments of food-related behaviors by segments of food desert and nonfood desert respondents of Guilford County. } \\
\hline NFD & \multicolumn{3}{|c|}{ FD } & \multicolumn{4}{|c|}{ NFD } \\
\hline & S I & S II & KW & AS & S I & S II & KW & AS \\
\hline I eat green salad & 121.61 & 140.28 & 4.07 & $0.04^{*}$ & 43.03 & 37.32 & 1.15 & 0.28 \\
\hline I eat fish & 108.03 & 146.38 & 15.67 & $0.00^{*}$ & 44.23 & 34.05 & 3.54 & 0.06 \\
\hline I eat fruit & 116.92 & 142.39 & 7.53 & $0.01^{*}$ & 44.42 & 33.55 & 4.02 & $0.05^{*}$ \\
\hline I eat lean meat & 119.34 & 141.3 & 5.21 & $0.02^{*}$ & 41.22 & 42.27 & 0.04 & 0.85 \\
\hline $\begin{array}{c}\text { I eat lunch dine at a café } \\
\text { restaurant }\end{array}$ & 150.81 & 127.18 & 5.78 & $0.02^{*}$ & 41.3 & 42.05 & 0.02 & 0.9 \\
\hline
\end{tabular}

KW represents Kruskal-Wallis H test, AS represents Asymp. Sig, * signifies significant at $5 \%$, SI \&SII represents Segment I \& Segment II mean ranks respectively.1: Never, 5: Always

Policies should expressly support a holistic approach that takes into account the psychometric characteristics of the target audience. All intervention programs should start with behaviors that are familiar to the target audience and involve them in the design of intervention programs. In this regard, intervention programs should build on lifestyle and behaviors that are familiar to residents rather than attempting to get them to learn wholly new behaviors at once. For example, since NFD respondents love to eat out where they are usually served more food of questionable quality; educating them on how to select healthy food off the menu and start with small portion size would be more effective in modifying their behavior rather than just asking them to stop eating out. And given that most restaurants now offer a menu with a wider variety of healthier choices this could be an effective ploy.

In the case of FD respondents that love to snack, a similar approach would entail training them to select and make healthier snack by paying attention to the ingredient content on the label and selecting healthy ingredients to prepare their own snacks. This is definitely a better approach than bombarding them with pedantic lessons on the need to eat healthy.

The study was limited by the small number of FD respondents and the method used to collect the data. In surveys, people tend to give socially desirable answers and may not have the detailed knowledge to answer the questions being asked. A qualitative follow up listening session could prove valuable in providing an in-depth explanation of patterns revealed by the numbers, as well as add insight not possible to get from the survey alone. Also, a larger sample size could provide a better comparison between FD residents and NFD residents of Guilford County and eliminate some of the difficulties we ran into during analysis.

\section{References}

1. Mclaughlin BN (2019) Greensboro, High Point top nationwide hunger list. p. 1-14.

2. Ver Ploeg M, Rahkovsky I (2016) Recent Evidence on the Effects of Food Store Access on Food Choice and Diet Quality. Amber Waves. (4): 1-8. 
3. Ghosh Dastidar M, Hunter G, Collins RL, Zenk SN, Cummins S, et al (2017) Does opening a supermarket in a food desert change the food environment? Heal Place 46: 249-256.

4. (2014) Why it takes more than a grocery store to eliminate a 'food desert'. p. 1-6.

5. Alert W (2019) Food-desert rescuer Renaissance Co-op in Greensboro is closing. p. 1-5.

6. Trimarchi BYM (2018) What's a food desert ? p. 1-5.

7. Whiteacre PT, Tsai P, Mulligan J (2009) The Public Health Effects of Food Deserts: Workshop Summary, Scitech Book News 34: 114

8. Thomas T, Gunden C (2017) Exploring the relationship among food-related values , food-related lifestyle and food-related behavior as lever for changing dietary habits of food desert residents 28(2): 67-72.

9. Grunert KG, Bruns $\varnothing$ K, Bredahl L, Bech AC (2001) Food-Related Lifestyle: A Segmentation Approach to European Food Consumers. In: Food, Peo ple and Society. Springer Berlin Heidelberg pp. 211-230.

10. Bruns $\varnothing$ K, Scholderer J, Grunert KG. Testing relationships between values and food-related lifestyle: results from two European countries. Appetite 43(2): 195-205.

11. Huang G, Grunert KG, Zhou Y, Krystallis A, Perrea T, Sørensen BT (2010) Is food-related lifestyle (FRL) able to reveal food consumption patterns in non-Western cultural environments? Its adaptation and application in urban China. Appetite 56(2): 357-367.

12. Scholderer J, Brunso K, Grunert KG (2002) Means-End Theory of Lifestyle-A Replication in the UK. Adv Consum Res 29: 551-557.
13. O’Neill M, Rebane D, Lester C (2004) Barriers to healthier eating in a disadvantaged community. Health Educ J 63(3): 220-228.

14. Trapp GSA, Hickling S, Christian HE, Bull F, Timperio AF, et al. (2015) Individual, Social, and Environmental Correlates of Healthy and Unhealthy Eating. Heal Educ Behav 42(6): 759-768.

15. Dubowitz T, Ghosh Dastidar M, Cohen DA, Beckman R, Steiner ED, et al. (2015) Diet and perceptions change with supermarket introduction in a food desert, but not because of supermarket use. Health Aff 34(11): 1858-1868.

16. Tabachnick BG, Fidell LS (2007)Using Multivariate Statistics Title: Using multivariate statistics.

17. Joseph F Hair, William C Black, Barry J Babin, Rolp E JF (2014) Seventh Edit ion Multivariate Data Analysis Hair Black Babin Anderson (Editor).

18. Mazzocchi M (2011) Statistics for Marketing and Consumer Research. Statistics for Marketing and Consumer Research. SAGE Publications Ltd.

19. Naresh K Malhotra (2010) Marketing Research- An Applied Orientation. pp.937.

20. Lusk JL, Briggeman BC (2009) Food values. Am J Agric Econ 91(1): 184196.

21. Bruns K, Grunert KG (1995) Development and Testing of a Cross-Culturally Valid Instrument: Food-Related Life Style. Adv Consum Res 22(1): 475-480.

22. Gunden C, Thomas T (2004) Capitalizing on the Relationship among Food-related Values, Food-related Lifestyle and Food-related Behavior for Promoting Healthy Dietary Behavior among Food Desert Residents. 